\title{
Association Between Arsenic Exposure and a Measure of Subclinical Sensory Neuropathy in Bangladesh
}

Danella M. Hafeman, ScB

Habibul Ahsan, MD, MMedSci

Elan D. Louis, MD, MS

Abu B. Siddique, MBBS

Vesna Slavkovich, MS

Zhongqi Cheng, PhD

Alexander van Geen, PhD

Joseph H. Graziano, PhD

\section{Learning Objectives}

- Describe, in this Bangladeshi population that has been chronically exposed to arsenic in drinking water, any associations between various measures of exposure and vibrotactile thresholds measured in the great toe and index finger (the primary outcome).

- Recall the degree of correlation, if any, between exposure to arsenic and several tasks of neurological function that served as secondary outcomes.

- Conclude whether the presence and concentration of manganese in drinking water influenced the association between arsenic exposure and signs of subclinical peripheral neuropathy.

\section{Abstract}

objectives: We examined the association between arsenic exposure and peripheral neuropathy in Bangladesh, where the population has been chronically exposed to arsenic in drinking water. Methods: We conducted a cross-sectional study of 137 subjects derived from a larger cohort. Exposure measures included individual water arsenic concentration, cumulative arsenic index, and urinary arsenic concentration taken at two time points (2001 and 2003). The primary outcome measurement was elevated vibrotactile threshold, as measured by a vibration sensitivity tester (Vibratron II). Results: Arsenic exposure was associated with elevated toe vibration threshold (TVT). Specifically, cumulative arsenic index and urinary arsenic (2001) were both significantly associated with elevated TVT $(\mathrm{P}=0.02$ and $\mathrm{P}=0.009$, respectively) after adjustment for age and gender. Conclusions: Increased arsenic exposure, as measured by both cumulative and urinary measures, was associated with evidence of subclinical sensory neuropathy. (J Occup Environ Med. 2005;47:778-784)

From the Mailman School of Public Health, Columbia University, New York, New York (Ms Hafeman, Dr Ahsan, Ms Slavkovich, Dr Graziano); Gertrude H. Sergievsky Center, College of Physicians and Surgeons, Columbia University, New York, New York (Dr Louis); Columbia University Arsenic Project, Araihazar, Bangladesh (Mr Siddique); and Lamont-Doherty Earth Observatory, Columbia University, New York, New York (Dr Cheng, Dr van Geen).

Supported by U.S. National Institute of Environmental Health Sciences Grants \# P42 ES10349 and \# P30 ES09089.

Address correspondence to Joseph H. Graziano, Mailman School of Public Health, 60 Haven Ave B-1, New York, NY 10032; E-mail: jg24@ columbia.edu.

Copyright ( $\odot$ by American College of Occupational and Environmental Medicine

DOI: 10.1097/01.jom.0000169089.54549.db hronic arsenic exposure is known to cause a wide variety of health effects, ${ }^{1-5}$ including peripheral neuropathy. $^{6-9}$ However, previous studies have used ecologic exposure variables instead of individual measures of arsenic exposure. Chronic arsenic exposure through drinking water is a major health hazard in many parts of the world. In Bangladesh, an estimated 50 million people are currently exposed to arsenic through this mechanism. ${ }^{10-12} \mathrm{Ar}$ senic exposure has been shown to be associated with cognitive delays in children in Bangladesh, ${ }^{13}$ implying effects on the central nervous system. However, controlled studies of the adverse effects on the peripheral nervous system have not previously been conducted in this setting.

The primary goals of this study were to: 1) establish a field method for further investigation of peripheral neuropathy in this population, and 2) collect preliminary data on the association between individual arsenic exposure measures and indicators of subclinical neuropathy. Our most valid outcome measure a priori was vibrotactile threshold, as quantified by a vibration sensitivity tester (Vibratron II).

\section{Materials and Methods}

\section{Study Design and Subjects}

The subjects recruited to participate in this study were residents of Araihazar, Bangladesh, and were between the ages of 20 and 50 years. All subjects were part of an ongoing prospective cohort study, Health Ef- 
fects of Arsenic Longitudinal Study (HEALS). As part of HEALS, a clinic was established to provide basic primary care services exclusively for 12,000 participants and their families; it is currently the chief source of care for these individuals.

Approximately 90 participants visit the clinic each day, either for their own care or to accompany a family member; an average of 5 of these individuals each day were selected randomly to participate in the study of peripheral neuropathy, yielding a total of 140 subjects recruited during an 8-week period. Exclusion criteria were thyroid disease (determined by physical examination for goiter), diabetes (determined by glucosuria), stroke (determined by self-report), and alcoholism (determined by selfreport). Two subjects $(1.4 \%)$ were excluded because of diabetes and one $(0.7 \%)$ because of stroke, leaving 137 subjects. The purpose of the study was explained to the participants, and informed consent was obtained in Bengali. This study was approved by the Columbia Presbyterian IRB and the Bangladesh Medical Research Council.

The basic study procedure consisted of a structured interview (conducted in Bengali by one of three research assistants) followed by a battery of neurological tests. These included vibrotactile threshold, tapping speed, grip strength, ankle reflex attenuation, and proprioception. These neurological tests were selected according to the distinguishing features of arsenic-induced neuropathy based on a review of the literature. $^{6,7,14}$ Development of the outcomes and training was conducted in association with a boardcertified neurologist (E.L.). All neurological tests were performed by one of the investigators (D.H.), who had been trained by a board-certified neurologist (E.L.), while a research assistant gave instructions in Bengali. After these tests, urine was collected and tested for the presence of glucose; separate aliquots were col- lected in acid-washed vessels and saved for further analysis. The entire procedure took approximately 20 to 30 minutes per subject.

\section{Exposure Assessment}

Four measures of arsenic exposure were obtained for each subject. Three measures of arsenic exposure (urinary arsenic, water arsenic, and cumulative arsenic index) were taken upon recruitment into the parent HEALS (2001). A fourth measure (urinary arsenic) was obtained at the time of the present study (2003). The mean difference between the two urine collection times was 2.0 years (SD, 0.4). Water manganese was also measured for 135 of the 137 subjects, using the water sample collected at the initial recruitment into HEALS.

Water samples were collected at the beginning of the cohort study as part of a survey of all wells in the study region. ${ }^{15}$ Field sample collection and laboratory analysis procedures are described in detail by van Geen et $\mathrm{al}^{15}$ and Cheng et al. ${ }^{16}$ In brief, samples were collected in 60-mL acid cleaned polyethylene bottles and 1-mL $7 \mathrm{~N}$ high purity $\mathrm{HCl}$ was added for preservation before shipping to the US (Lamont Doherty Earth Observatory of Columbia University) for analysis. For As concentration determination the samples were diluted 1:10 using a matrix modifier consisting of $2 \%$ $\mathrm{HNO}_{3}$ and 50-ppm nickel and then analyzed by graphite furnace atomic absorption spectrometry (GFAAS, Hitachi Z-8200). The estimated standard deviation for individual measurements was $\sim 7 \mu \mathrm{g} / \mathrm{L}$. Mn concentrations were determined by high resolution inductively coupled plasma mass spectrometry (HR ICPMS, Axiom SC), with a detection limit of $0.1 \mu \mathrm{g} / \mathrm{L}$.

Urine samples were collected in $50-\mathrm{mL}$ acid washed plastic tubes at the time of the interview and immediately placed in a cooler by field staff. All urine samples were stored in coolers in the field, frozen at $-20^{\circ} \mathrm{C}$ within 8 hours, and sub- sequently shipped to Columbia University on dry ice. ${ }^{17}$ The samples collected for the present study were handled in a comparable fashion. The urinary arsenic concentration assays were performed by graphite furnace atomic absorption spectrometry methods using a Perkin-Elmer Analyst 600 graphite furnace, as described. ${ }^{18}$ All measurements are expressed as micrograms of arsenic per gram creatinine, which was measured using a colorimetric Sigma Diagnostics Kit (Sigma, St. Louis, MO).

The cumulative arsenic index (CAI) was calculated by multiplying water arsenic concentration by the estimated amount of water drunk per year times the number of years the well had been in use by each study participant. Daily water consumption was estimated by multiplying the number of glasses of water consumed per day times the volume of a typical glass. If the participant had changed wells and arsenic concentration was known for the previous well, this information was also taken into account. The median duration of exposure information for our participants was nine years.

\section{Primary Outcome Measurements}

Vibrotactile Threshold Testing. The Vibratron II (Physitemp, Inc., Clifton, NJ) has been used to study the neurological effects of arsenic dust exposure, ${ }^{7}$ diabetic neuropathy, ${ }^{19}$ organophosphate neuropathies, ${ }^{20,21}$ and cisplatin-induced peripheral neuropathy. ${ }^{22}$ We used a well-established forced choice testing algorithm to assess the cutaneous vibration threshold at $100 \mathrm{~Hz}^{23,24}$ Briefly, the subjects were told that one of two rods was vibrating, and they were to identify this rod. If their assessment was correct, the vibration amplitude was decreased approximately $10 \%$ on the log scale; if a mistake was made, amplitude was increased by the same percentage. After five mistakes, an average was taken of the five lowest correct and five mistakes (removing the highest 
and lowest values) to obtain the vibration threshold in log microns of displacement (vibration units, vu). Measurements were taken on the subjects' right index fingers and left great toes; these values are referred to as index vibration threshold (IVT) and toe vibration threshold (TVT), respectively.

\section{Secondary Outcome Measurements}

Tapping Speed. Subjects were asked to tap repeatedly for a duration of 10 seconds with the index finger of their dominant hand, followed by their nondominant hand. The number of taps was counted. The sum of these two trials was used as the outcome.

Grip Strength. Subjects were asked to squeeze a hand-held device (Baseline 220-lb Smedley Spring Dynamometer) as hard as possible with their dominant hand. The mean force generated over three trials was recorded. Next, they completed the same task with their non-dominant hand. The sum of the dominant and non-dominant measures was calculated and used as the outcome measure.

Ankle Reflex. Ankle reflexes were taken in a cross-legged position while subjects were distracted with a simple task. On each side, the examiner elicited the ankle reflex five times (10 total). Subjects were given a score according to the number of reflexes that the examiner could elicit (out of a possible ten attempts). For the purposes of analysis, the bottom quartile (a score of six or less out of ten) was compared to the top three quartiles (a score of seven or more).

Proprioception. The Romberg sign and pronator drift were assessed by assigning all subjects a score of absent, equivocal, or present for both of these measures. To assess the Romberg sign, the participant was asked to stand with his or her feet together and eyes closed. Loss of balance indicated a positive sign, whereas marked swaying was categorized as equivocal. Pronator drift was assessed by asking the patient to hold his or her arms outstretched with palms facing upward and eyes closed. Moderate movement of the palms was categorized as equivocal, while marked pronation indicated a positive sign.

\section{Statistical Analysis}

Outcome data are reported as means \pm standard deviations (vibrotactile threshold, grip strength, and tapping speed) or as frequencies (ankle reflex and proprioception). Data were first analyzed according to quartiles of water, cumulative, and urinary arsenic exposure. Continuous data were displayed graphically as boxplots, and analysis of variance tests were performed to determine differences in means according to arsenic quartile. For the frequency data, $\chi^{2}$ tests were conducted to detect a difference in frequency across arsenic quartile.

Next, linear regression analyses were used to examine the association between arsenic exposure and continuous outcomes (vibration threshold measures, grip strength, and tapping speed). Assumptions of linear regression were tested and confirmed: vibrotactile threshold, grip strength, and tapping speed were found to be continuous, normally distributed variables. Linear regression analyses were then run for the three measurements of arsenic (water, cumulative, urinary) as both dummy (tertiles) and continuous variables. All results were adjusted for age and gender. Because manganese exposure has been shown to be associated with both arsenic exposure and neurological outcomes, ${ }^{13,16,25-27}$ we also assessed water manganese exposure as a potential confounder of this association. In addition, linear regression analyses were used to examine the effect of water manganese without adjustment for arsenic exposure (controlling for age and gender).
Logistic regression analyses were used to examine the association of exposure with reflex attenuation and proprioception. Reflex attenuation was a dichotomous variable $(1=$ if subjects had a sum of 6 or less out of 10 , and $0=$ if they received a score of 7 or more out of 10). Proprioception was considered abnormal (proprioception $=1$ ) if both the Romberg sign and the pronator drift were equivocal or either was abnormal; otherwise, it was considered normal (proprioception $=0$ ). Results were adjusted for age and gender. As above, results were also subsequently adjusted for water manganese. Finally, logistic regression analyses were used to examine the effect of water manganese without adjustment for arsenic exposure (controlling for age and gender).

\section{Results}

Table 1 shows the subjects' demographic characteristics, as well as the mean values for the four exposure measures and primary outcome variables (IVT and TVT). Arsenic exposure among these subjects has decreased between the time of recruitment into the parent cohort in 2001 and the current study in 2003, likely as a result of intervention strategies that have been described elsewhere. $^{28}$ The mean urinary arsenic concentration in 2001 was 326.3 (SD, 308), whereas the mean in 2003 was 252.4 (SD, 185) $\mu \mathrm{gAs} / \mathrm{mgCr}$ $(P=0.01$; Table 1$)$. The correlation coefficients among the four exposure measures were high, but did not approach one. In particular, the correlation coefficient between urinary arsenic concentration taken during the present study versus during cohort recruitment was 0.657 .

The average water manganese concentration in this study sample was $1.19 \mathrm{mg} / \mathrm{L}$ (SD, 0.83), more than twice the WHO standard of 0.5 $\mathrm{mg} / \mathrm{L}$. The correlation between water arsenic and water manganese concentrations approached significance (Spearman $r=0.16, P=0.06$ ). However, there was no association 
between manganese water concentration and cumulative arsenic (Spearman $r=0.13, P=0.14)$, total urinary arsenic concentration (2001) (Spearman $r=0.004, P=0.96$ ), or total urinary arsenic concentration (2003) (Spearman $r=0.04, P=$ $0.61)$. TVT values were increased in the highest quartile of urinary arsenic (2001) and water arsenic, but in an analysis of variance, these differences were not significant (Fig. 1).

Table 2 shows the linear regression analyses for the associations between TVT and measure of arsenic exposure, while controlling for age and gender. Age was an important negative confounder because it was negatively associated with both urinary arsenic (2001) in this study sample (Spearman $r=-0.227, P<$ 0.01 ) and positively associated with TVT (Spearman $r=0.377, P<$ $0.001)$. The continuous measures of total urinary arsenic (2001) and CAI (2001) were both significant predictors of TVT score. Therefore, for every $50 \mu \mathrm{gAs} / \mathrm{mgCr}$ increase in total urinary arsenic (2001), there is an estimated 0.02 increase in TVT score. (An increase of $1455 \mu \mathrm{gAs} /$ $\mathrm{mgCr}$ in urinary arsenic (within the range of exposure) would be associated with an elevation in TVT score approximately equal to one standard deviation $(0.55 \mathrm{vu})$. The urinary $(P=0.07)$ and water $(P=0.06)$ tertile coefficients also approached statistical significance (Table 2).

Adjustment for water manganese did not appreciably change these beta estimates, indicating that the exposure was not a confounder for the association between arsenic and TVT in this study. Water manganese was also not a significant predictor of TVT, with or without adjustment for arsenic exposure.

Table 3 shows the linear regression analyses for the IVT. Although TVT and IVT were correlated $\left(\mathrm{r}^{2}=\right.$ $0.158, P<0.001)$, the association between measures of arsenic exposure and IVT did not approach statistical significance. No association was found between any measure- ment of arsenic and grip strength, tapping speed, proprioception measures, or reflex measures. Water manganese was not a significant predictor of any of the outcomes tested, with or without adjustment for arsenic exposure.

\section{Discussion}

The findings of this study demonstrate an association between chronic arsenic exposure and a subclinical loss of vibratory sensation in the lower extremities. Specifically, con-
TABLE 1

Descriptive Characteristics of Study Population $(n=137)$

\begin{tabular}{|c|c|c|}
\hline Age (years), mean (SD) & 33.7 & $(7.0)$ \\
\hline 20 to 29 years, $n(\%)$ & 43 & (31) \\
\hline 30 to 39 years, $n(\%)$ & 64 & $(47)$ \\
\hline 40 to 50 years, $n(\%)$ & 29 & $(21)$ \\
\hline \multicolumn{3}{|l|}{ Gender } \\
\hline Male, $n(\%)$ & 71 & $(52)$ \\
\hline Female, $n(\%)$ & 66 & $(48)$ \\
\hline \multicolumn{3}{|l|}{ Exposure measures, mean (SD) } \\
\hline Urinary As (2001), $\mu \mathrm{g} / \mathrm{mgCr}$ & 326.3 & $(307.5)$ \\
\hline Water As (2001), $\mu \mathrm{g} / \mathrm{L}$ & 115.0 & $(140.0)$ \\
\hline CAI (2001), $\mu \mathrm{g}$ & 1271.1 & $(2085.2)$ \\
\hline Urinary As (2003), $\mu \mathrm{g} / \mathrm{mgCr}$ & 252.4 & $(185.4)$ \\
\hline Water Mn (2001), mg/L & 4.86 & (1.19) \\
\hline \multicolumn{3}{|l|}{ TVT (vibration units) } \\
\hline Mean (SD) & \multicolumn{2}{|c|}{$1.20(0.54)$} \\
\hline Geometric mean & \multicolumn{2}{|c|}{1.08} \\
\hline \multicolumn{3}{|l|}{ IVT (vibration units) } \\
\hline Mean (SD) & \multicolumn{2}{|c|}{$1.01(0.45)$} \\
\hline Geometric mean & \multicolumn{2}{|c|}{0.92} \\
\hline
\end{tabular}

a.
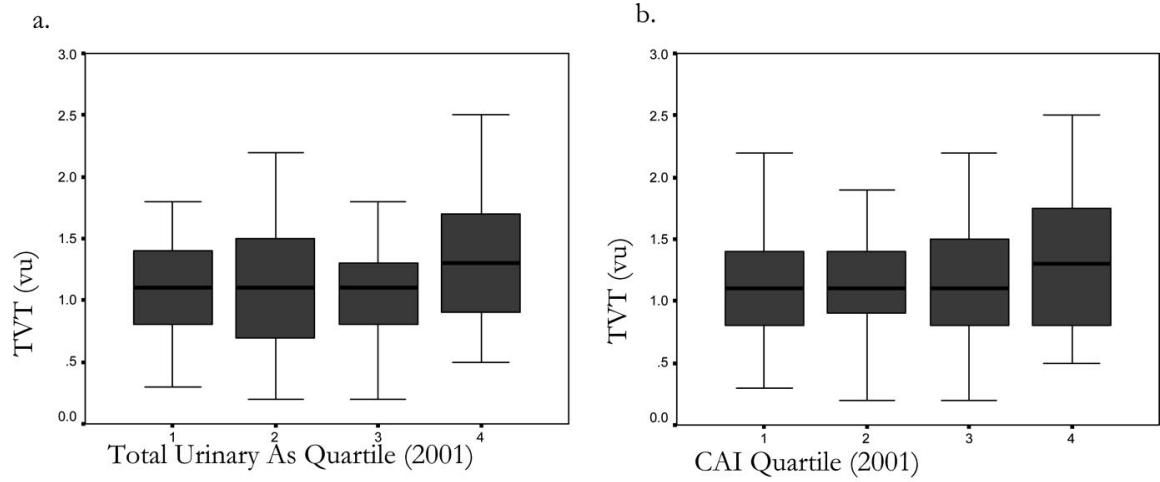

d.
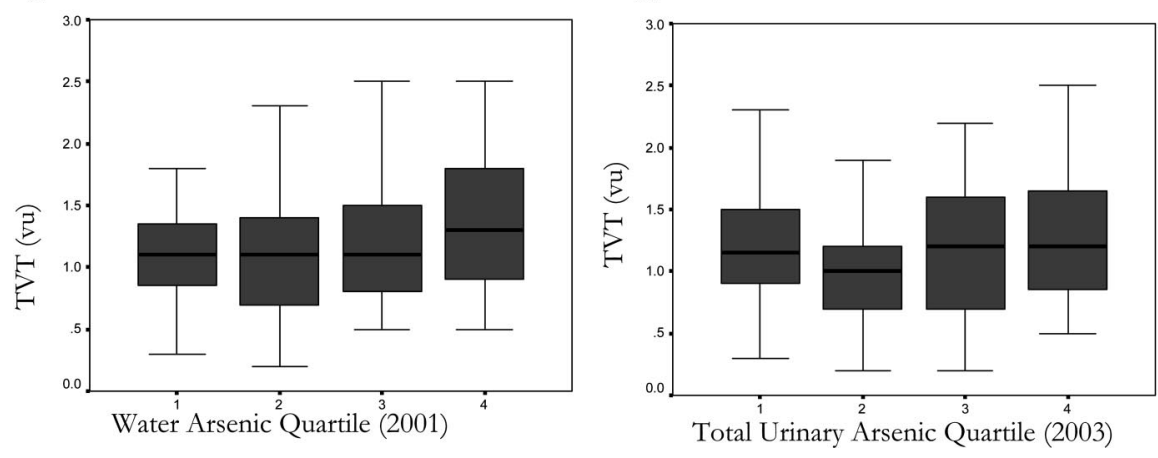

Fig. 1. Boxplots of TVT versus arsenic exposure quartile. Plots (a) to (c) show that TVT increases with various measures of arsenic exposure, especially for the highest quartile of exposure. There is no evident trend of TVT according to urinary total as quartile (d; 2003). 


\section{TABLE 2}

Linear Regression Analyses Examining the Association Between TVT and Measures of Arsenic Exposure*

\begin{tabular}{lcc} 
& B (SE) & $\boldsymbol{P}$ Value \\
\hline Continuous (per 50 units) & $0.019(0.007)$ & 0.009 \\
Urinary As (2001) & $0.025(0.016)$ & 0.11 \\
Water As (2001) & $0.510^{-3}\left(1.08 \times 10^{-3}\right)$ & 0.02 \\
CAl (2001) & $0.014(0.012)$ & 0.26 \\
Urinary As (2003) & - & \\
Tertiles: & $0.019(0.105)$ & - \\
Urinary As (2001) & $0.197(0.107)$ & 0.86 \\
25.5-148.7 & - & - \\
149.1-325.5 & -5.07 \\
332.6-1736.9 & $0.203(0.105)$ & 0.99 \\
Water As (2001) & - & 0.06 \\
$5-23$ & $-0.009(0.109)$ & - \\
$25-125$ & $0.129(0.109)$ & 0.41 \\
$129-743$ & - & 0.24 \\
CAl (2001) & - \\
$2.9-159.1$ & $-0.039(0.107)$ & 0.71 \\
$159.5-843.7$ & $-0.138(0.106)$ & 0.20 \\
$953.3-11482.5$ & &
\end{tabular}

* Estimates adjusted for age and gender.

\section{TABLE 3}

Linear Regression Analyses Examining the Association Between IVT and Measures of Arsenic Exposure*

\begin{tabular}{ccc} 
& B (SE) & $\boldsymbol{P}$ Value \\
\hline Continuous (per 50 units) & & \\
Urinary As (2001) & $-3.64 \times 10^{-3}\left(6.40 \times 10^{-3}\right)$ & 0.57 \\
Water As (2001) & $-0.013(0.014)$ & 0.35 \\
CAl (2001) & $-0.008(0.011)$ & 0.97 \\
Urinary As (2003) & - & 0.42 \\
Tertiles: & $-0.106(0.093)$ & \\
Urinary As (2001) & $-0.068(0.095)$ & - \\
25.5-148.7 & - & 0.26 \\
149.1-325.5 & $0.058(0.097)$ & 0.48 \\
332.6-1736.9 & $-0.013(0.095)$ & - \\
Water As (2001) & - & 0.55 \\
$5-23$ & $-0.087(0.097)$ & 0.89 \\
$25-125$ & $0.038(0.097)$ & - \\
$129-743$ & - & 0.38 \\
CAl (2001) & 0.70 \\
$2.9-159.1$ & $-0.039(0.093)$ & - \\
$159.5-843.7$ & $0.129(0.093)$ & 0.68 \\
$953.3-11482.5$ & & 0.17 \\
Urinary As (2003) & & \\
36.5-147.0 & & \\
$150.8-270.5$ & & \\
$271.4-975.4$ & &
\end{tabular}

* Estimates adjusted for age and gender.

tinuous measures of cumulative and urinary arsenic measures assessed 3 years before the study, but not current urinary arsenic, were associated with an increase in vibrotactile threshold in the lower extremity.

Peripheral neuropathy caused by chronic arsenic exposure has been described as a primarily sensory neuropathy, ${ }^{29}$ characterized by the dying back of axons ${ }^{30}$ followed by secondary demyelination. ${ }^{6}$ Long neurons have been shown to be preferentially affected and are also the slowest to recover. ${ }^{6}$ Evidence indicates that long-term cumulative arsenic exposure is a more important predictor of neuropathy than short-term fluctuations ${ }^{8}$ and that symptoms of neuropathy last for years after the exposure has ceased. ${ }^{31}$ In fact, Lakergvist et $\mathrm{al}^{8}$ found that symptoms actually increased during a 5-year period following reduction of exposure in copper smelter workers.

Based on these characteristics of arsenic-induced neuropathy, the findings presented here are not surprising. First, we found that arsenic exposure was associated with increased vibrotactile threshold, a sign of subclinical sensory neuropathy. Second, we found this association with respect to the arsenic measurements ascertained 3 years before the outcome measurement (2001), but not the urinary arsenic determined at the time of the present study (2003). Although the correlation between these values was high, there were appreciable changes in arsenic exposures. Because peripheral neuropathy has a long latency time, the initial exposure measures (2001) were probably a better indicator of the relevant construct. During the interim between the two arsenic exposure measurements, significant decreases in exposure have occurred in our study population due, at least in part, to an education campaign and well-testing; the 2001 measures were taken prior to these interventions (following a relatively more stable exposure period) and probably reflect more accurately arsenic exposure during earlier years. Third, CAI, but not water arsenic, was associated with subclinical sensory neuropathy. Although water arsenic is a simple measure of exposure at a single point in time, $\mathrm{CAI}$ is an estimated index of cumulative exposure over many years. The fact that this historical 
measure (but not water arsenic) was associated with the outcome corroborates the hypothesis that cumulative exposure is a more important predictor of outcome than short-term measures.

We found a strong association between urinary arsenic and TVT measures but not IVT measures. Because the literature indicates that long fibers are preferentially affected over shorter fibers, it is not surprising that TVT, but not IVT, was affected. However, given the nature of the current study protocol, another possibility must be considered. The IVT was taken after a short training trial, followed by the TVT trial. It is possible that this study population required a longer training trial, and that other factors led to measurement error that masked the effect of arsenic.

We did not find an association between measures of arsenic exposure and any of the other outcomes tested. There are two likely explanations for these null findings. First, arsenic exposure has been shown to cause a primarily sensory neuropathy, so it is possible that the outcomes assessing motor function (such as grip strength and tapping speed) would not be affected. Second, nondifferential misclassification might have obscured associations between exposure and other outcomes assessing sensory function (such as proprioception and reflex attenuation). Both proprioception and reflex assessment were assessed qualitatively, and these observations may have lacked the accuracy to detect the small differences necessary.

Although there is evidence that chronic arsenic exposure is associated with peripheral neuropathy, the literature is far from consistent on the issue. In particular, several studies have failed to show an effect of chronic arsenic exposure on electrophysiological abnormalities (ie, increased nerve conduction velocities), even in the presence of symptomatic neuropathy. 7,32 On the other hand, one study found evidence of decreased nerve conduction velocities, but only modest symptomatic neuropathy and no EMG changes in workers exposed to arsenic at a copper smelter. ${ }^{8}$ Finally, Kreiss et al $^{33}$ found no effect of arsenic exposure on either nerve conduction velocities or prevalence of symptomatic neuropathy.

The most consistent findings have come from studies assessing a predominantly sensory neuropathy utilizing sensitive and quantitative instruments. Gerr et $\mathrm{al}^{7}$ used the Vibratron II to determine vibrotactile threshold changes associated with arsenic dust exposures in Georgia and found significant differences between unexposed and exposed subjects on both IVT and TVT measures. Another study in Taiwan found that current perception thresholds were higher in exposed compared to unexposed individuals. ${ }^{14}$ These findings provide evidence for an arsenic-induced subclinical sensory peripheral neuropathy, consistent with the results of the current study. However, the above studies used ecological measures of arsenic exposure, and thus could not determine the quantitative effect of individual exposure to arsenic. In the current study, we determined the vibrotactile thresholds of subjects with a wide range of arsenic exposure. The results from this study indicate that there is a relationship between individual arsenic exposure and vibrotactile threshold.

Our ascertainment of this outcome should be interpreted in the context of the following weakness. Our study participants were not randomly sampled from the population, but rather consisted of people who came into the study clinic for their own care or to accompany a family member. However, the clinic is the main primary care source for the 12,000 member cohort, and the reasons for the subjects' visits were not related to neurological disorders or arsenicosis. Thus it is reasonable to assume that individuals who came to the clinic for care were not systemati- cally different from those who did not in a way that would bias results.

Taken in the context of previous studies, the current findings add to the evidence that chronic arsenic exposure via a contaminated water source is associated with an indicator of subclinical sensory peripheral neuropathy. In summary, our results indicate that there is a significant association between urinary and cumulative arsenic exposures and measures of subclinical peripheral neuropathy. However, the true doseresponse relationship remains to be characterized.

\section{Acknowledgments}

The authors would like to acknowledge study participants for their willingness to participate in this study. In addition, we would like to thank A. H. M. Reazuddin, Benay Kumar Datta, and the rest of our field staff for their dedication and hard work.

\section{References}

1. Tseng WP, Chu HM, How SW, et al. Prevalence of skin cancer in an endemic area of chronic arsenicism in Taiwan. J Natl Cancer Inst. 1968;40:453-463.

2. Smith AH, Goycolea M, Haque R, Biggs ML. Marked increase in bladder and lung cancer mortality in a region of Northern Chile due to arsenic in drinking water. Am J Epidemiol. 1998;147:660-669.

3. Tseng WP. Effects and dose-response relationships of skin cancer and blackfoot disease with arsenic. Environ Health Perspect. 1977;19:109-119.

4. Morales KH, Ryan L, Kuo TL, Wu MM, Chen CJ. Risk of internal cancers from arsenic in drinking water. Environ Health Perspect. 2000;108:655-661.

5. Tseng $\mathrm{CH}$. An overview on peripheral vascular disease in blackfoot diseasehyperendemic villages in Taiwan. Angiology. 2002;53:529-537.

6. Feldman RG, Niles CA, Kelly-Hayes M, et al. Peripheral neuropathy in arsenic smelter workers. Neurology. 1979;29: 939-944.

7. Gerr FE, Letz R, Ryan PB, Green RC. Neurological effects of environmental exposure to arsenic in dust and soil among humans. Neurotoxicology. 2000; 21:475-487.

8. Lagerkvist BJ, Zetterlund B. Assessment of exposure to arsenic among smelter workers: a five-year follow-up. Am J Ind Med. 1994;25:477-488.

9. Tseng $\mathrm{CH}$, Chong $\mathrm{CK}$, Tseng $\mathrm{CP}$, et al. 
Long-term arsenic exposure and ischemic heart disease in arseniasis-hyperendemic villages in Taiwan. Toxicol Lett. 2003; 137:15-21.

10. MacDonald R. Providing clean water: lessons from Bangladesh. BMJ. 2001; 322:626-627.

11. Smith AH, Lingas EO, Rahman M. Contamination of drinking-water by arsenic in Bangladesh: a public health emergency. Bull World Health Organ. 2000; 78:1093-1103.

12. British Geological Survey. Phase 2 groundwater studies of arsenic contamination in Bangladesh. British Geological Survey, Nottingham; 2001.

13. Wasserman GA, Liu X, Parvez F, et al. Water arsenic exposure and children's intellectual function in Araihazar, Bangladesh. Environ Health Perspect. 200; 112:1329-1333.

14. Tseng $\mathrm{CH}$. Abnormal current perception thresholds measured by neurometer among residents in blackfoot diseasehyperendemic villages in Taiwan. Toxicol Lett. 2003;146:27-36.

15. van Geen A, Zheng Y, Versteeg R, et al. Spatial variability of arsenic in 6000 tube wells in a $25 \mathrm{~km}^{2}$ area of Bangladesh. Water Resources Res. 2003;39:11401150.

16. Cheng Z, Zheng Y, Mortlock R, Van Geen A. Rapid multi-element analysis of groundwater by high-resolution inductively coupled plasma mass spectrometry. Anal Bioanal Chem. 2004;379: 512-518.

17. Ahsan H, Perrin M, Rahman A, et al. Associations between drinking water and urinary arsenic levels and skin lesions in Bangladesh. J Occup Environ Med. 2000; 42:1195-1201.
18. Nixon DE, Mussmann GV, Eckdahl SJ, Moyer TP. Total arsenic in urine: palladium-persulfate vs nickel as a matrix modifier for graphite furnace atomic absorption spectrophotometry. Clin Chem. 1991;37:1575-1579.

19. Sosenko JM, Gadia MT, Natori N, et al. Neurofunctional testing for the detection of diabetic peripheral neuropathy. Arch Intern Med. 1987;147:1741-1744.

20. London L, Thompson ML, Capper W, Myers JE. Utility of vibration sense testing for use in developing countries: comparison of extinction time on the tuning fork to vibration thresholds on the Vibratron II. Neurotoxicology. 2000;21:743752.

21. London L, Nell V, Thompson ML, Myers JE. Effects of long-term organophosphate exposures on neurological symptoms, vibration sense and tremor among South African farm workers. Scand J Work Environ Health. 1998;24:18-29.

22. Roberts JA, Jenison EL, Kim K, ClarkePearson D, Langleben A. A randomized, multicenter, double-blind, placebocontrolled, dose-finding study of ORG 2766 in the prevention or delay of cisplatin-induced neuropathies in women with ovarian cancer. Gynecol Oncol. 1997;67: 172-177.

23. Gerr FE, Hershman D, Letz R. Vibrotactile threshold measurement for detecting neurotoxicity: reliability and determination of age- and height-standardized normative values. Arch Environ Health. 1990;45:148-154.

24. Gerr FE, Letz R. Reliability of a widely used test of peripheral cutaneous vibration sensitivity and a comparison of two testing protocols. Br J Ind Med. 1988;45: 635-639.
25. Kondakis XG, Makris N, Leotsinidis M, Prinou M, Papapetropoulos T. Possible health effects of high manganese concentration in drinking water. Arch Environ Health. 1989;44:175-178.

26. Mergler D, Baldwin M, Belanger S, et al. Manganese neurotoxicity, a continuum of dysfunction: results from a community based study. Neurotoxicology. 1999;20: 327-342.

27. Beuter A, Edwards R, deGeoffroy A, Mergler D, Hundnell K. Quantification of neuromotor function for detection of the effects of manganese. Neurotoxicology. 1999;20:355-466.

28. Van Geen A, Ahsan H, Horneman AH, et al. Promotion of well-switching to mitigate the current arsenic crisis in Bangladesh. Bull World Health Organ. 2002; 80:732-737.

29. Jenkins RB. Inorganic arsenic and the nervous system. Brain. 1966;89:479498.

30. Windebank AJ. Specific inhibition of myelination by lead in vitro; comparison with arsenic, thallium, and mercury. Exp Neurol. 1986;94:203-212.

31. LeQuesne PM, McLeod JG. Peripheral neuropathy following a single exposure to arsenic. Clinical course in four patients with electrophysiological and histological studies. J Neurol Sci. 1977;32:437451.

32. Mazumder DN, Das Gupta J, Chakraborty $\mathrm{AK}$, et al. Environmental pollution and chronic arsenicosis in south Calcutta. Bull World Health Organ. 1992;70:481-485.

33. Kreiss K, Zack MM, Landrigan PJ, et al. Neurologic evaluation of a population exposed to arsenic in Alaskan well water. Arch Environ Health. 1983;38:116-121. 